\title{
SCHOTTKY LEVELS AND THERMODYNAMIC CONTRIBUTIONS OF LIGHT LANTHANIDE SESQUISULFIDES HAVING THE $\mathrm{Th}_{3} \mathrm{P}_{4}$ STRUCTURE*
}

JOHN B. GRUBER

Departments of Physics and Chemistry, Portland State University, Portland, OR 97207 (U.S.A.)

R. BURRIEL and EDGAR F. WESTRUM, JR.

Department of Chemistry, University of Michigan, Ann Arbor, MI 48109(U.S.A.)

P. E. PALMER and B. J. BEAUDRY

Ames Laboratory, Iowa State University, Ames, IA 50011 (U.S.A.)

(Received March 3, 1983)

\section{Summary}

Heat capacity measurements were made by adiabatic calorimetry over the range $7-350 \mathrm{~K}$ on $\gamma$ phase preparations of four lanthanide sesquisulfides, and the heat capacities were resolved into lattice, magnetic, Schottky and other components. The entropy at $298.15 \mathrm{~K}$ for $\mathrm{La}_{2} \mathrm{~S}_{3}$ which is written as $S^{\circ} / R$ is 19.51 while values of $S^{\circ}-S^{\circ}(7 \mathrm{~K})$ for $\mathrm{Ce}_{2} \mathrm{~S}_{3}, \mathrm{Nd}_{2} \mathrm{~S}_{3}, \mathrm{Gd}_{2} \mathrm{~S}_{3}$ and $\mathrm{Dy}_{2} \mathrm{~S}_{3}$ are 21.34, 22.38, 20.05 and 22.58 respectively. IR and visible optical spectra of $\mathrm{La}_{2} \mathrm{~S}_{3}, \mathrm{Ce}_{2} \mathrm{~S}_{3}$, $\mathrm{Nd}_{2} \mathrm{~S}_{3}$ and $\mathrm{Dy}_{2} \mathrm{~S}_{3}$ and lattice sum crystal field splitting calculations for $\mathrm{Nd}_{2} \mathrm{~S}_{3}$, $\mathrm{Ce}_{2} \mathrm{~S}_{3}$ and $\mathrm{Dy}_{2} \mathrm{~S}_{3}$ are compared with Raman scattering data and Schottky contributions derived from calorimetry.

\section{Introduction}

The lanthanide sesquisulfides $\operatorname{Ln}_{2} \mathrm{~S}_{3}$ manifest unusual magnetic and electronic behavior as a consequence of their (4f $)^{n}$ states [1-3]. The study of these phenomena by calorimetric, spectroscopic and magnetic techniques leads to an elucidation and resolution of their energetic spectra. To date relatively few heat capacity data have been reported [4-7] and they have not been interpreted in specific detail between 20 and $300 \mathrm{~K}$ other than to establish the possible occurrence of magnetic ordering or structural phase transitions. A successful interpretation of the observed phenomena taking account of crystal

* Paper presented at the Sixteenth Rare Earth Research Conference, The Florida State University, Tallahassee, FL, U.S.A., April 18-21, 1983. 
field effects and available lower temperature heat capacity data $[7,8]$ has permitted evaluation of the thermodynamic functions and resolution of the Schottky contributions together with correlation with IR and Raman scattering data presented here and in the literature [8-11].

\section{Experimental details}

\subsection{Sample provenance and characterization for heat capacity studies}

The sulfides were prepared at the Ames Laboratory, Iowa State University, by direct combination of high purity elements produced in the Laboratory in a manner similar to that described by Gschneidner et al. [12]. The quartz ampoules containing the reacted elements were opened and the sulfide was ground and sieved to 200 mesh powder, cold pressed into pellets and heated under a dynamic $\mathrm{H}_{2} \mathrm{~S}$ atmosphere for $24-48 \mathrm{~h}$ at $1450-1500^{\circ} \mathrm{C}$.

Debye-Scherrer X-ray patterns contained only lines of the $\gamma$ phase b.c.c. $\mathrm{Th}_{3} \mathrm{P}_{4}$ structure. The final compositions were determined by chemical analysis to be stoichiometric within the experimental uncertainty. The clarity of the solution in $6 \mathrm{~N} \mathrm{HCl}$ indicates the absence of trace oxysulfide impurities. All samples used in this study gave clear solutions when dissolved.

\subsection{Automated adiabatic calorimetry}

The data were obtained using the mark X calorimetric cryostat, which is an improved (by the addition of a guard shield surrounding the adiabatic shield) version of the mark II cryostat described in ref. 13 where the relevant operating techniques were also given. The operation of the instruments and the acquisition of heat capacity data from about 7 to $350 \mathrm{~K}$ was computer assisted. Temperatures and other accurate experimental quantities are referred to standards provided by the National Bureau of Standards.

\subsection{Schottky heat capacity contributions}

Resolution of Schottky contributions from heat capacity data on lanthanide compounds requires an accurate determination of the much larger "lattice" contribution. The heat capacity volume-weighted lattice approximation technique employed for $\mathrm{Ce}_{2} \mathrm{~S}_{3}, \mathrm{Nd}_{2} \mathrm{~S}_{3}$ and $\mathrm{Dy}{ }_{2} \mathrm{~S}_{3}$ has been used successfully for several other groups of lanthanide compounds [14] and involves linear interpolation between diamagnetic $\mathrm{La}_{2} \mathrm{~S}_{3}$ and $\mathrm{Gd}_{2} \mathrm{~S}_{3}$ with the magnetic contribution deleted. It should be noted that the mole throughout is based on the formula written as $\operatorname{Ln}_{2} S_{3}$ or 2 mol of lanthanide.

\subsection{Optical spectroscopy: samples, technique and measurements}

Samples used in the investigation of the IR spectra were prepared by passing purified $\mathrm{H}_{2} \mathrm{~S}$ gas over graphite boats containing finely ground $\mathrm{Ln}_{2} \mathrm{O}_{3}$ powder (minimum purity, $99.99 \%$ ) at $850-1300^{\circ} \mathrm{C}$. Analysis of the X-ray crystallography patterns revealed that the dark red-brown to black powders had the high temperature $\gamma$ phase $\mathrm{Th}_{3} \mathrm{P}_{4}$ b.c.c. structure. Chemical analysis of the powder indicated that the material was stoichiometric. 
IR spectra of powdered mull samples were recorded between 250 and $2500 \mathrm{~cm}^{-1}$ using a Perkin-Elmer 301 spectrometer. Samples were cooled to $15 \mathrm{~K}$ and to $90 \mathrm{~K}$ using a conduction Dewar filled with liquid helium and liquid nitrogen respectively. The measurements were performed by J. R. Henderson, M. Muramoto and J. B. Gruber between 1967 and 1970 as part of a comprehensive research project to investigate the optical, magnetic and electrical properties of the lanthanide sesquisulfides. Only part of these investigations has been reported $[3,9,10]$.

\section{Results and discussion}

\subsection{Spectroscopic properties}

The IR spectra between 250 and $2500 \mathrm{~cm}^{-1}$ contain a number of very strong bands which are broad even at $15 \mathrm{~K}\left(25-50 \mathrm{~cm}^{-1}\right)$. Tracings of these recordings have appeared earlier [3,9]. There is a band with structure near $270 \mathrm{~cm}^{-1}$ in $\mathrm{La}_{2} \mathrm{~S}_{3}$ and near $275 \mathrm{~cm}^{-1}$ in $\mathrm{Ce}_{2} \mathrm{~S}_{3}$. A second band is found at $300 \mathrm{~cm}^{-1}$ and at $310 \mathrm{~cm}^{-1}$ for $\mathrm{La}_{2} \mathrm{~S}_{3}$ and $\mathrm{Ce}_{2} \mathrm{~S}_{3}$ respectively. A relatively narrow peak of moderate strength (half the absorbance of bands found lower in energy) is observed at approximately $358 \mathrm{~cm}^{-1}$ in $\mathrm{Ce}_{2} \mathrm{~S}_{3}$. This peak, which is not found in the $\mathrm{La}_{2} \mathrm{~S}_{3}$ spectrum, may be due to an electronic (magnetic dipole) transition within the ground state manifold ${ }^{2} \mathrm{~F}_{5 / 2}$ of the $\mathrm{Ce}^{3+}(4 \mathrm{f})^{1}$ ion. Since the point group symmetry of $\mathrm{Ce}^{3+}$ in the $\mathrm{R}_{2} \mathrm{~S}_{3}$ lattice is $S_{4}$, the ground state manifold $J=\frac{5}{2}$ is split into three Kramers doublets. Earlier measurements and interpretation of magnetic susceptibility confirm the ground state level to be a doublet in $\mathrm{Ce}_{2} \mathrm{~S}_{3}$ $[15,16]$. Absorption above $380 \mathrm{~cm}^{-1}$ is too broad for any structure to be identified.

The spectra of powdered mull samples were investigated between 30 and $300 \mathrm{~cm}^{-1}$ using a Beckman FS-720 Fourier interference spectrophotometer and a conduction Dewar filled with liquid nitrogen. Bands approximately $15-25$ $\mathrm{cm}^{-1}$ wide at half-maximum absorption were obtained at $60(63) \mathrm{cm}^{-1}, 120(123)$ $\mathrm{cm}^{-1}, 170(175) \mathrm{cm}^{-1}, 230(235) \mathrm{cm}^{-1}$ and $270(275) \mathrm{cm}^{-1}$ for $\mathrm{La}_{2} \mathrm{~S}_{3}\left(\mathrm{Ce}_{2} \mathrm{~S}_{3}\right)$. The $80 \mathrm{~K}$ spectrum of $\mathrm{Ce}_{2} \mathrm{~S}_{3}$ at $185 \mathrm{~cm}^{-1}$ shows a shoulder not observed in the $\mathrm{La}_{2} \mathrm{~S}_{3}$ spectra which may be due to a second electronic transition within the ${ }^{2} \mathrm{~F}_{5 / 2}$ manifold. Otherwise both spectra appear roughly the same with the $\mathrm{Ce}_{2} \mathrm{~S}_{3}$ spectrum shifted slightly to the high energy side of the $\mathrm{La}_{2} \mathrm{~S}_{3}$ spectrum. Both the IR and the Raman spectral data are summarized in Table 1, and the data for $\mathrm{Ce}_{2} \mathrm{~S}_{3}$ are compared graphically in Fig. 1 with those derived from the calorimetric data of Fig. 2. An equally excellent correlation obtains for $\mathrm{Nd}_{2} \mathrm{~S}_{3}$.

\subsection{Thermophysical properties}

The calorimetrically derived Schottky contribution to the heat capacity of $\mathrm{Ce}_{2} \mathrm{~S}_{3}$ is displayed as the full curve in Fig. 1. The entropy at $298.15 \mathrm{~K}$ for $\mathrm{La}_{2} \mathrm{~S}_{3}$, which is written as $S^{\circ} / R$, is 19.51 while values of $S^{\circ}-S^{\circ}(7 \mathrm{~K})$ for $\mathrm{Ce}_{2} \mathrm{~S}_{3}, \mathrm{Nd}_{2} \mathrm{~S}_{3}$, $\mathrm{Gd}_{2} \mathrm{~S}_{3}$ and $\mathrm{Dy}_{2} \mathrm{~S}_{3}$ are $21.34,22.38,20.05$ and 22.58 respectively.

Recent reports by various Russian groups, including the results of room 
TABLE 1

IR and Raman spectra for several lanthanide sesquisulfides

\begin{tabular}{|c|c|c|c|c|}
\hline \multicolumn{2}{|l|}{$L a_{2} S_{3}$} & \multirow{2}{*}{$\begin{array}{l}\mathrm{Ce}_{2} S_{3} \\
\operatorname{IR}\left(\mathrm{cm}^{-1}\right)\end{array}$} & \multicolumn{2}{|l|}{$\mathrm{Nd}_{2} \mathrm{~S}_{3}$} \\
\hline$I R\left(\mathrm{~cm}^{-1}\right)$ & $\operatorname{Raman}\left(\mathrm{cm}^{-1}\right)$ & & $I R\left(\mathrm{~cm}^{-1}\right)$ & $\operatorname{Raman}\left(\mathrm{cm}^{-1}\right)$ \\
\hline $60^{\mathrm{z}}$ & $65^{\mathrm{b}}$ & $63^{\mathrm{a}}$ & $64^{\mathrm{a}}$ & - \\
\hline - & $85^{b}$ & - & $76^{\mathrm{a} . \mathrm{e}}$ & $80^{b}$ \\
\hline $120^{\mathrm{a}}, 127^{\mathrm{d}}$ & $122^{b}$ & $123^{\mathrm{a}}$ & $124^{\mathrm{a}}$ & - \\
\hline- & - & - & $140^{\mathrm{a}, \mathrm{c}}, 150^{\mathrm{a} \cdot \mathrm{c}}$ & - \\
\hline $170^{\mathrm{a}}, 180^{\mathrm{d}}$ & $185^{b}$ & $175(185)^{9}$ & $180^{\mathrm{a} \cdot \mathrm{c}}, 200^{\mathrm{d}}$ & $192^{b}$ \\
\hline $230^{\mathrm{a}}, 230^{\mathrm{d}}$ & $232^{b}$ & $235^{\mathrm{a}}$ & $235^{\mathrm{a}}, 240^{\mathrm{d}}$ & $237^{6}$ \\
\hline $270^{\circ}, 270^{\mathrm{d}}$ & $273^{\mathrm{b}}$ & $275^{*}$ & $278^{4}$ & $287^{\circ}$ \\
\hline $300^{\mathrm{d}}$ & $300^{d}$ & $310^{\mathrm{a}}$ & - & - \\
\hline - & - & $358^{\mathrm{a}}$ & $385^{\mathrm{a}}$ & - \\
\hline
\end{tabular}

From ref. 17 (spectra recorded at $90 \mathrm{~K}$ ).

${ }^{b}$ From ref. 11 (spectra recorded at room temperature).

' From ref. 18 (spectra recorded at $90 \mathrm{~K}$ ).

${ }^{\mathrm{d}}$ From ref. 19 (spectra recorded at room temperature).
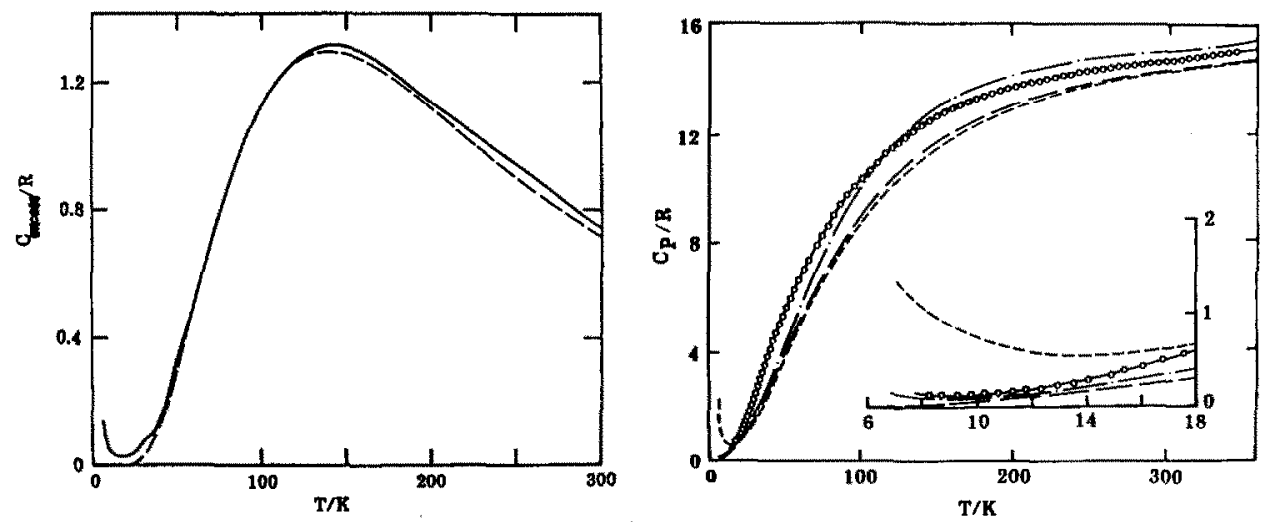

Fig. 1. Comparison of the calorimetric Schottky contributions, i.e. $C_{p}\left(\mathrm{Ce}_{2} \mathrm{~S}_{3}\right)-\left\{0.39 C_{p}\left(\mathrm{Gd}_{2} \mathrm{~S}_{3}\right.\right.$. corrected for magnetic ordering) $\left.-0.61 C_{p}\left(\mathrm{La}_{2} \mathrm{~S}_{3}\right)\right\}$ (-) with spectroscopic contributions calculated from the Stark levels $\left(0,185\right.$ and $\left.358 \mathrm{~cm}^{-1}\right)(--)$.

Fig. 2. Molar heat capacities: experimental points are shown for $\mathrm{La}_{2} \mathrm{~S}_{3}(-)$ to indicate their approximate density for all samples; the curves for $\mathrm{Ce}_{2} \mathrm{~S}_{3}(-\cdots), \mathrm{Nd}_{2} \mathrm{~S}_{3}(---)$ and $\mathrm{Gd}_{2} \mathrm{~S}_{3}(\cdots)$ are shown without the experimental data to avoid confusion.

temperature Raman scattering spectra of $\mathrm{La}_{2} \mathrm{~S}_{3}$ and $\mathrm{Dy}_{2} \mathrm{~S}_{3}$ [11], are in agreement with the vibrational spectra observed in the unpublished work of Gruber et al. [10]. While assignments of vibrational and electronic transitions to the spectra are difficult to ascertain from the optical data alone, it is hoped that future crystal field splitting calculations and the interpretation of unpublished magnetic susceptibility data will further clarify the details of the Stark splitting of the ground state manifolds of all rare earth ions in the sesquisulfide lattice. 


\section{Acknowledgments}

We wish to thank R. Hofer and R. Z. Bachman, Analytical Services Group, Ames Laboratory, Iowa State University, for the chemical analyses of samples used to make heat capacity measurements, and J. R. Henderson and D. Johnson, McDonnell Douglas Astronautics Company, Santa Monica, CA, for preparing samples used to make the IR spectra measurements. We thank Professor Magda El-Fass, Xiao-Xia Ma and W. A. Plautz for assisting in the evaluation of the heat capacities and thermodynamic functions.

The portion of this work done at the University of Michigan was supported in part by the Structural Chemistry and Chemical Thermodynamics Program of the National Science Foundation under Grant CHE-8007977.

\section{References}

1 G. H. Dieke, Spectroscopic Properties of Rare Earth Ions, Wiley-Interscience,-New York, 1965.

2 B. R. Judd, Operator Techniques in Atomic Spectroscopy, McGraw-Hill, New York, 1963.

3 J. R. Henderson, M. Muramoto, E. Loh and J. B. Gruber, J. Chem. Phys., 47 (1967) 3347.

4 E. G. King and W. W. Weller, Low temperature heat capacities and entropies at $298.15 \mathrm{~K}$ of cerium monosulfide, cerium sesquisulfide and thorium disulfide, Rep. USMB-R1-5485, 1959 (U.S. Bureau of Mines).

5 V.V. Nogteva, I. E. Paukov and E. I. Yarembash, Russ. J. Phys. Chem., 43(1969) 1312.

6 V. V. Tikhonov and I. A. Smirnov, Sov. Phys. - Solid State, 13 (1972) 2296.

7 J. C. Ho, S. M. A. Taher, G. B. King, J. B. Gruber, B. J. Beaudry and K. A. Gschneidner, Jr., J. Phys. (Paris), Colloq. C6, $8(1978) 840$.

8 S. M. A. Taher, J. C. Ho and J. B. Gruber, J. Chem. Phys., 76 (1982) 609.

9 J. R. Henderson, M. Muramoto, J. B. Gruber and R. Menzel, J. Chem. Phys., 52 (1970) 2311.

10 J. B. Gruber, J. R. Henderson and M. Muramoto, Rep. DAC-59368P, October 1966; Rep. DAC-605317R, June 1968 (McDonnell Douglas Astronautics Company, Santa Monica, CA) (unpublished).

11 T. G. Arkatova, V. P. Zhuze, M. G. Kariss, A. A. Kamarzin, A. A. Kukharskî̂, B. A. Mikhailon and A. I. Skelykh, Sov. Phys. - Solid State, 21 (1979) 1979.

12 K. A. Gschneidner, Jr., B. J. Beaudry, T. Takeshita, S. S. Eucker, S. M. A. Taher, J. C. Ho and J. B. Gruber, Phys. Rev, B, 24 (1981) 7187.

13 E. F. Westrum, Jr., J. Chem. Educ., 39 (1962) 443.

E. F. Westrum, Jr., G. T. Furukawa and J. P. McCullough, in J. P. McCullough and D. W. Scott (eds.), Experimental Thermodynamics, Vol. 1, Butterworths, London, 1968, Chap. 4, p. 133.

14 E. F. Westrum, Jr., J. Chem. Thermodyn., 15(1983), in the press.

15 A. T. Starovoitov, V. I. Ozhogin, G. M. Loginov and V. M. Sergeeva, Zh. Eksp. Teor. Fiz., 57(1969) 791 (Sov. Phys. -JETP, 30 (1970) 433).

16 G. Becker, J. Feldhaus, K. Westerholt and S. Methfessel, J. Magn. Magn. Mater., 6 (1977) 14.

17 J. A. Henderson, M. Muramoto and J. B. Gruber, unpublished data, 1969.

18 J. R. Henderson, M. Muramoto, J. B. Gruber and R. Menzel, J. Chem. Phys., 52 (1970) 2311.

19 V. P. Zakharov and G. V. Lashkarev, Preparation and Investigation of the Properties of Rare Earth Compounds, Soviet Physics Publishers, Kiev, 1975, p. 82. 